\title{
New passivity criteria for discrete-time neural networks with leakage and time-varying delays
}

\author{
Wei Kang ${ }^{1,2, a}$, Shouming Zhong ${ }^{1, b}$ and Yunli $\mathrm{HaO}^{2, \mathrm{c}}$ \\ ${ }^{1}$ School of Mathematical Sciences, University of Electronic Science and Technology of China, \\ Chengdu 611731, PR China \\ ${ }^{2}$ School of Information Engineering, Fuyang Normal University, Fuyang \\ 236041, PR China \\ akangwei0830@163.com, 'bhongsm@uestc.edu.cn, chaoyunli9116@163.com
}

Keywords: Passivity; Discrete-time neural networks; Leakage delay; Time-varying delay.

\begin{abstract}
In this paper, the problem of passivity of discrete-time neural networks with leakage and time-varying delays is investigated. By constructing a novel Lyapunov-Krasovskii functional and reciprocally convex method, some sufficient passivity conditions are obtained in the forms of linear matrix inequalities. In order to illustrate the effectiveness of the proposed results, a numerical example is presented.
\end{abstract}

\section{Introduction}

It is well known that the neural networks are very important since they have been widely applied in various areas, such as combinatorial optimization, pattern recognition and so on [1-2]. On the other hand, time delay is unavoidable in real systems and they often bring about instability, oscillation or divergence in neural networks. Therefore, a lot of interesting results on stability analysis for delayed system have been obtained in the literature [3-7].

It should be mentioned that many neural networks are focused on continuous-time cases. However, discrete-time systems are more suitable for computer computation and simulation. Thus, it is necessary and important to research the dynamics of discrete-time systems, and a large number of results have been achieved [4-5]. For instance, Kang et al. [4] proposed the stability criteria for the discrete-time system with time varying delay by means of Lyapunov-Krasovskill functional theory , reciprocally convex approach and linear matrix inequalities technique.

Passivity is a significant concept that represents input-output feature of dynamic systems. In the past several decades, the passivity theory has found successful applications in various areas such as signal processing, stability and fuzzy control. Thus, the passivity problem for time-delay neural networks has received considerable attention [1-2, 6]. In [1-2], the problem of passivity for continuous-time neural networks with time-delay was studied by using the linear matrix inequality technique. In [6], some sufficient passivity conditions have been obtained for discrete-time stochastic neural networks with time-varying delays in terms of linear matrix inequality by utilizing the Lyapunov functional method. However, there are still a lot of rooms for further decreasing the conservatism.

Motivated by the above discussion, the problem of passivity for discrete-time neural networks with leakage and time-varying delays is studied in this paper. By employing a new Lyapunov-Krasovskii functional and reciprocally convex approach, some improved conditions are obtained in terms of linear matrix inequalities. Finally, a numerical example is provided to illustrate the effectiveness of the proposed method.

\section{Problem statement and preliminaries}

In this paper, we consider the following discrete-time neural networks with time-varying delays: 


$$
\left\{\begin{array}{c}
x(k+1)=C x(k-\delta)+A f(x(k))+B f(x(k-\tau(k)))+u(k) \\
y(k)=f(x(k)), \quad i=1,2, \cdots n \\
x(j)=\psi(j), \quad j=-\rho,-\rho+1, \cdots,-1, \quad \rho=\max \left(\delta, \tau_{M}\right)
\end{array}\right.
$$

Where $x(k)=\left[x_{1}(k), x_{2}(k), \cdots, x_{n}(k)\right]^{T}$ is the neural state vector of the system, $C=\operatorname{diag}\left(c_{1}, c_{2}, \cdots, c_{n}\right)$ is a selt-feedback connection matrix. $A=\left(a_{i j}\right)_{n \times n}, B=\left(b_{i j}\right)_{n \times n}$ are the connection weight and the delayed connection weight matrices. $f(x(k))=\left[f_{1}\left(x_{1}(k)\right), f_{2}\left(x_{2}(k)\right), \cdots, f_{n}\left(x_{n}(k)\right)\right]^{T}$ is the nonlinear activation function, $u(k)$ is the input, $y(k)$ represents the output of the network. $\tau(k)$ denotes the discrete time-varying delay and satisfies $\tau_{m} \leq \tau(k) \leq \tau_{M}, k \in N$, where $\tau_{m}, \tau_{M}$ are known positive integers.

Assumption 1 The transformed neuron acfivation functions satisfies:

$$
l_{j}^{-} \leq \frac{f_{j}\left(s_{1}\right)-f_{j}\left(s_{2}\right)}{s_{1}-s_{2}} \leq l_{j}^{+}, f_{j}(0)=0, j=1,2, \cdots, n
$$

Where $l_{j}^{-}, l_{j}^{+}$are known real constants.

To obtain our results, we need introduce the following definition and lemma.

Definition 1[6] The system is said to be passive if there exists a scalar $\gamma>0$ satisfying

$$
2 \sum_{i=0}^{k_{0}} y^{T}(i) u(i) \geq-\gamma \sum_{i=0}^{k_{0}} u^{T}(i) u(i)
$$

For all $k_{0} \in N$ and under the zero initial condition.

Lemma 1 [2, 6] For any matrices $\left[\begin{array}{cc}T & N \\ \bullet & T\end{array}\right]>0, \tau_{m}, \tau_{M}, \tau(k)$ satisfying $\tau_{m} \leq \tau(k) \leq \tau_{M}$, and vector function $x(k+\cdot) \rightarrow R^{n}$, such that the sums concerned are well defined, then

$$
-\tau_{M m} \sum_{i=k-\tau_{M}}^{k-\tau_{m}-1} \eta^{T}(i) T \eta(i) \leq \alpha^{T}(k)\left[\begin{array}{ccc}
-2 T+N^{T}+N & T-N^{T} & T-N \\
\bullet & -T & N \\
\bullet & \bullet & -T
\end{array}\right] \alpha(k)
$$

Where $\alpha(k)=\left[x^{T}(k-\tau(k)), x^{T}\left(k-\tau_{m}\right), x^{T}\left(k-\tau_{M}\right)\right]^{T}$.

\section{Main results}

For the sake of simplicity of matrix and vector representation, $e_{i} \in R^{9 n \times n}(i=1,2, \cdots, 9)$ are defined as block entry matrices (for example $e_{1}=\left[\begin{array}{lllllllll}I & 0 & 0 & 0 & 0 & 0 & 0 & 0 & 0\end{array}\right]^{T}$ ). The other notations are defined as

$$
\begin{aligned}
L_{1}=\operatorname{diag}\left(l_{1}^{-} l_{1}^{+}, \cdots, l_{n}^{-} l_{n}^{+}\right), L_{2}=\operatorname{diag}\left(\frac{l_{1}^{-}+l_{1}^{+}}{2}, \cdots, \frac{l_{n}^{-}+l_{n}^{+}}{2}\right), \eta(k)=x(k+1)-x(k), \tau_{M m}=\tau_{M}-\tau_{m} \\
\xi^{T}(k)=\left[x^{T}(k), x^{T}(k-\delta), x^{T}\left(k-\tau_{m}\right), x^{T}(k-\tau(k)), x^{T}\left(k-\tau_{M}\right), f^{T}(x(k)), f^{T}(x(k-\tau(k))),\right. \\
\left.\eta^{T}(k), u^{T}(k)\right]
\end{aligned}
$$

Theorem 1 For given integers $\tau_{m}, \tau_{M}$, the discrete-time neural networks (1) is passive, if there exist matrices $P>0, R_{i}>0(i=1,2,3), Q=\left[\begin{array}{cc}Q_{11} & Q_{12} \\ \bullet & Q_{22}\end{array}\right]>0, Z_{1}>0, Z_{2}>0$, two positive diagonal matrices $S_{i}=\operatorname{diag}\left(s_{1 i}, s_{2 i}, \cdots, s_{n i}\right), i=1,2$, matrices $U_{1}, U_{2}, U_{3}, N$, and the scalar $\gamma>0$, such the following matrix inequalities hold:

$$
\left[\begin{array}{cc}
Z_{2} & N \\
\cdot & Z_{2}
\end{array}\right]>0, \quad \Omega_{1}+\Omega_{2}<0 .
$$


Where

$$
\begin{aligned}
\Omega_{1}= & {\left[e_{2} C+e_{6} A+e_{7} B+e_{9}\right] P\left[e_{2} C^{T}+e_{6} A^{T}+e_{7} B^{T}+e_{9}\right]^{T}+e_{1}\left(R_{1}+R_{2}+R_{3}\right) e_{1}^{T}-e_{2} R_{1} e_{2}^{T} } \\
& -e_{3} R_{2} e_{3}^{T}-e_{5} R_{3} e_{5}^{T}+\left(\tau_{M m}+1\right) \Pi_{1} Q \Pi_{1}^{T}-\left(\tau_{M m}+1\right) \Pi_{2} Q \Pi_{2}^{T}+e_{8}\left(\tau_{m}^{2} Z_{1}+\tau_{m}^{2} Z_{2}\right) e_{8}^{T} \\
& -\Pi_{3}\left[\begin{array}{cc}
-Z_{1} & Z_{1} \\
\bullet & -Z_{1}
\end{array}\right] \Pi_{3}^{T}-\Pi_{4}\left[\begin{array}{ccc}
-2 Z_{2}+N^{T}+N & Z_{2}-N^{T} & Z_{2}-N \\
\bullet & -Z_{2} & N \\
\bullet & \bullet & -Z_{2}
\end{array}\right] \Pi_{4}^{T} \\
& -\Pi_{1}\left[\begin{array}{cc}
S_{2} L_{1} & -S_{2} L_{2} \\
\bullet & S_{2}
\end{array}\right] \Pi_{1}^{T}-\Pi_{2}\left[\begin{array}{cc}
S_{2} L_{1} & -S_{2} L_{2} \\
\bullet & S_{2}
\end{array}\right] \Pi_{2}^{T} \\
\Omega_{2}= & {\left[e_{1} U_{1}+e_{2} U_{2}+e_{8} U_{3}\right]\left[e_{2}(C-I)^{T}+e_{6} A^{T}+e_{7} B^{T}+e_{9}-e_{8}\right]^{T} } \\
\Pi_{1}= & {\left[e_{1}, e_{5}\right], \Pi_{2}=\left[e_{4}, e_{6}\right], \Pi_{3}=\left[e_{1}, e_{3}\right], \Pi_{4}=\left[e_{4}, e_{3}, e_{5}\right] }
\end{aligned}
$$

Proof Consider the following Lyapunov-Krasovskii functional: $V(k)=\sum_{i=1}^{4} V_{i}(k)$

$$
\begin{aligned}
& V_{1}(k)=x^{T}(k) P x(k) \\
& V_{2}(k)=\sum_{i=k-\delta}^{k-1} x^{T}(i) R_{1} x(i)+\sum_{i=k-\tau_{m}}^{k-1} x^{T}(i) R_{2} x(i)+\sum_{i=k-\tau_{M}}^{k-1} x^{T}(i) R_{3} x(i) \\
& V_{3}(k)=\sum_{i=k-\tau(k)}^{k-1}\left(\begin{array}{c}
x(i) \\
f(x(i))
\end{array}\right)^{T} Q\left(\begin{array}{c}
x(i) \\
f(x(i))
\end{array}\right)+\sum_{j=-\tau_{M}}^{-\tau_{m}-1} \sum_{i=k+j}^{k-1}\left(\begin{array}{c}
x(i) \\
f(x(i))
\end{array}\right)^{T} Q\left(\begin{array}{c}
x(i) \\
f(x(i))
\end{array}\right) \\
& V_{4}(k)=\tau_{m} \sum_{j=-\tau_{m}}^{-1} \sum_{i=k+j}^{k-1} \eta^{T}(i) Z_{1} \eta(i)+\tau_{M m} \sum_{j=-\tau_{M}}^{-\tau_{m}-1} \sum_{i=k+j}^{k-1} \eta^{T}(i) Z_{2} \eta(i)
\end{aligned}
$$

Then, taking the differences of $V_{i}(k)$ along the trajectories of the system (1), it yields

$$
\begin{aligned}
\begin{aligned}
\Delta V_{1}(k)= & x^{T}(k+1) P x(k+1)-x^{T}(k) P x(k) \\
= & {[C x(k-\delta)+A f(x(k))+B f(x(k-\tau(k)))+u(k)]^{T} P } \\
& {[C x(k-\delta)+A f(x(k))+B f(x(k-\tau(k)))+u(k)]-x^{T}(k) P x(k) } \\
\Delta V_{2}(k)= & x^{T}(k)\left(R_{1}+R_{2}+R_{3}\right) x(k)-x^{T}(k-\delta) R_{1} x(k-\delta)-x^{T}\left(k-\tau_{m}\right) R_{2} x\left(k-\tau_{m}\right) \\
& -x^{T}\left(k-\tau_{M}\right) R_{3} x\left(k-\tau_{M}\right) \\
\Delta V_{3}(k)= & \left.\left(\tau_{M m}+1\right)\left[\begin{array}{c}
x(k) \\
f(x(k))
\end{array}\right)^{T} Q\left(\begin{array}{c}
x(k) \\
f(x(k))
\end{array}\right)-\left(\begin{array}{c}
x(k-\tau(k)) \\
f(x(k-\tau(k)))
\end{array}\right)^{T} Q\left(\begin{array}{c}
x(k-\tau(k)) \\
f(x(k-\tau(k)))
\end{array}\right)\right] \\
\Delta V_{4}(k)= & \eta^{T}(k)\left(\tau_{m}^{2} Z_{1}+\tau_{M m}^{2} Z_{2}\right) \eta(k)-\tau_{m} \sum_{i=k-\tau_{m}}^{k-1} \eta^{T}(i) Z_{1} \eta(i)-\tau_{M m} \sum_{i=k-\tau_{M}}^{k-1} \eta^{T}(i) Z_{2} \eta(i)
\end{aligned}
\end{aligned}
$$

By Jensen inequality and Lemma 1, we can get

$$
\begin{aligned}
& -\tau_{m} \sum_{i=k-\tau_{m}}^{k-1} \eta^{T}(l) Z_{1} \eta(l) \leq\left(\begin{array}{c}
x(k) \\
x\left(k-\tau_{m}\right)
\end{array}\right)^{T}\left[\begin{array}{cc}
-Z_{1} & Z_{1} \\
Z_{1} & -Z_{1}
\end{array}\right]\left(\begin{array}{c}
x(k) \\
x\left(k-\tau_{m}\right)
\end{array}\right) \\
& -\tau_{M m} \sum_{i=k-\tau_{M}}^{k-\tau_{m}-1} \eta^{T}(i) Z_{2} \eta(i) \leq \alpha^{T}(k)\left[\begin{array}{ccc}
-2 Z_{2}+N^{T}+N & Z_{2}-N^{T} & Z_{2}-N \\
\bullet & -Z_{2} & N \\
\bullet & \bullet & -Z_{2}
\end{array}\right] \alpha(k)
\end{aligned}
$$


Where $\alpha(k)=\left[x^{T}(k-\tau(k)), x^{T}\left(k-\tau_{m}\right), x^{T}\left(k-\tau_{M}\right)\right]^{T}$

From Assumption 1, for the diagonal matrices $S_{k}=\operatorname{diag}\left(s_{1 k}, s_{2 k}, \cdots, s_{n k}\right), k=1,2$, we can receive the following inequalities:

$$
\begin{aligned}
& -\left(\begin{array}{c}
x(k) \\
f(x(k))
\end{array}\right)^{T}\left[\begin{array}{cc}
S_{1} L_{1} & -S_{1} L_{2} \\
\bullet & S_{1}
\end{array}\right]\left(\begin{array}{c}
x(k) \\
f(x(k))
\end{array}\right) \geq 0 \\
& -\left(\begin{array}{c}
x(k-\tau(k)) \\
f(x(k-\tau(k)))
\end{array}\right)^{T}\left[\begin{array}{cc}
S_{2} L_{1} & -S_{2} L_{2} \\
\bullet & S_{2}
\end{array}\right]\left(\begin{array}{c}
x(k-\tau(k)) \\
f(x(k-\tau(k)))
\end{array}\right) \geq 0
\end{aligned}
$$

On the other hand, for any matrices $U_{1}, U_{2}, U_{3}$ with appropriate dimensions, we can get

$$
\begin{aligned}
& 2\left[x^{T}(k) U_{1}+x^{T}(k-\delta) U_{2}+\eta^{T}(k) U_{3}\right] \\
& \quad[(C-I) x(k-\delta)+A f(x(k))+B f(x(k-\tau(k)))+u(k)-\eta(k)]=0
\end{aligned}
$$

Therefore, from (5) to ( 13), we have

$$
\Delta V(k)-2 y^{T}(k) u(k)-\gamma u^{T}(k) u(k) \leq \xi^{T}(k)\left[\Omega_{1}+\Omega_{2}\right] \xi(k)
$$

From (3), we can conclude that

$$
\Delta V(k)-2 y^{T}(k) u(k)-\gamma u^{T}(k) u(k) \leq 0
$$

Then, we have

$$
2 \sum_{i=0}^{k_{0}} y^{T}(i) u(i) \geq \sum_{i=0}^{k_{0}} \Delta V(i)-\gamma \sum_{i=0}^{k_{0}} u^{T}(i) u(i), \quad \sum_{i=0}^{k_{0}} \Delta V(i)=V\left(k_{0}+1\right)-V(0) \geq 0
$$

So, we can get

$$
2 \sum_{i=0}^{k_{0}} y^{T}(i) u(i) \geq-\gamma \sum_{i=0}^{k_{0}} u^{T}(i) u(i) \text {, for all } k_{0} \in N \text {. }
$$

The proof is completed.

\section{Numerical example}

Example 1 Consider the system (1) with the following parameters

$$
C=\left[\begin{array}{cc}
0.5 & 0 \\
0 & 0.6
\end{array}\right], A=\left[\begin{array}{cc}
0.001 & 0 \\
0 & 0.005
\end{array}\right], B=\left[\begin{array}{cc}
-0.1 & 0.01 \\
-0.2 & -0.1
\end{array}\right] \text {. }
$$

The activation functions are taken as

$$
f_{1}(x)=f_{2}(x)=\tanh (x) .
$$

For this example, in simulation let

$$
x(0)=[0.3,-0.2]^{T}, u(k)=\left[\sin \left(\frac{\pi k}{2}\right), \cos \left(\frac{\pi k}{2}\right)\right]^{T}, \tau(k)=6+4 \sin \left(\frac{\pi k}{2}\right), \delta=0,
$$

the state trajectories without input $u(k)$ and with $u(k)$ can be seen in Figs 1 and 2 .

\section{Conclusions}

In this paper, the problem of passivity analysis for discrete-time neural networks with leakage and time-varying delay has been investigated. The presented sufficient conditions are based on the Lyapunov method and the reciprocally convex technique. Finally, a numerical example is given to show the effectiveness of the proposed criteria. It should be worth mentioning that the proposed way can be extensively applicable in many other areas. 


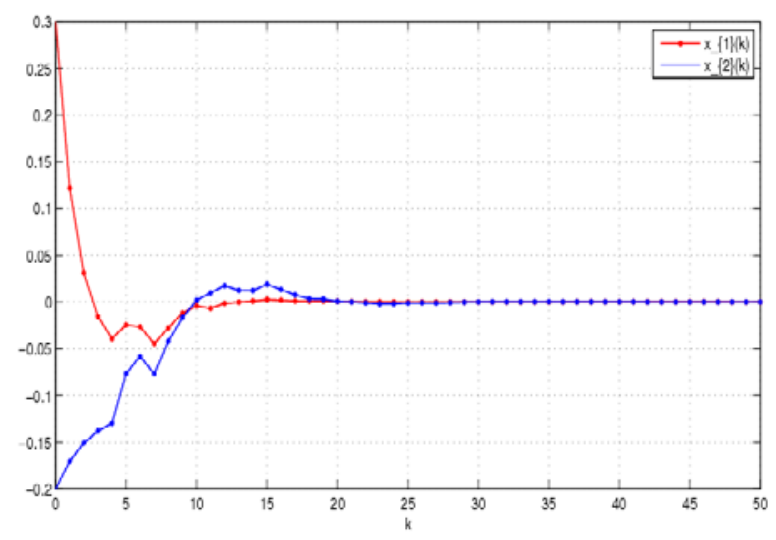

Figure1

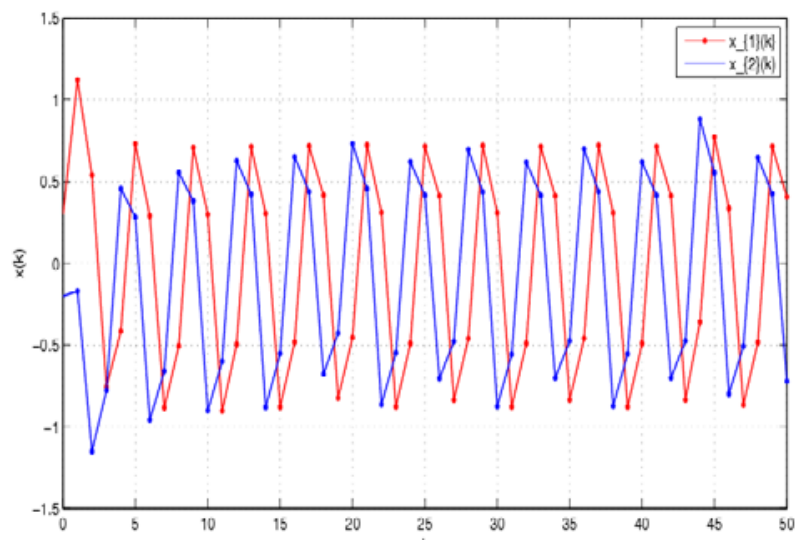

Figure2

\section{Acknowledgements}

This work was financially supported by the Natural Science Foundation of the Anhui Higher Education Institutions of China (KJ2016A555, KJ2016A625), the national science research project of FuYang normal University (2016FSKJ07) and Program for Excellent Young Talents in University of Anhui province (gxyq2017158).

\section{References}

[1] H. Zeng , Y. He, M. Wu, H. Xiao, Improved conditions for passivity of neural networks with a time-varying delay. IEEE Trans On Cybernetics 44, 785-792, 2014.

[2] Z. Wu, P. Shi, H. Su, J. Chu, Stability and dissipativity analysis of static neural networks with time delay. IEEE Trans. Neural Netw. Learn. Syst. 23, 199-210, 2014.

[3] PG. Park, JW. Ko, C.Jeong, Reciprocally convex approach to stability of systems with time-varying delays. Automatica. 47, 235-238, 2011.

[4] W. Kang, S. Zhong, K. Shi, J. Cheng, Finite-time stability for discrete-time system with time-varying delay and nonlinear perturbations. ISA Transactions. 60, 63-70, 2016.

[5] W. Kang, S. Zhong, J. Cheng, Relaxed passivity conditions for discrete-time stochastic delayed neural networks. International Journal of Machine Learning and Cybernetics.7, 205-216, 2016.

[6] A. Seuret, F. Gouaisbaut, Wirtinger-based integral inequality: application to time-delay systems. Automatica, 49, 2860-2866, 2013.

[7 ] WI. Lee, PG. Park, Second-order reciprocally convex approach to stability of systems with interval time-varying delays.Appl. Math. Comput, 229, 245-253,2014 